\title{
Criminologie
}

\section{Une expérience de court terme selon Carkhuff au service de probation du Québec}

\section{Nicole Morin Laurendeau}

Volume 12, numéro 2, 1979

Probation : aide ou contrainte ?

URI : https://id.erudit.org/iderudit/017108ar

DOI : https://doi.org/10.7202/017108ar

Aller au sommaire du numéro

Éditeur(s)

Les Presses de l'Université de Montréal

ISSN

0316-0041 (imprimé)

1492-1367 (numérique)

Découvrir la revue

Citer cet article

Morin Laurendeau, N. (1979). Une expérience de court terme selon Carkhuff au service de probation du Québec. Criminologie, 12(2), 58-77.

https://doi.org/10.7202/017108ar d'utilisation que vous pouvez consulter en ligne.

https://apropos.erudit.org/fr/usagers/politique-dutilisation/ 


\section{UNE EXPERIENCE DE COURT TERME SELON CARKHUFF AU SERVICE DE PROBATION DU QUÉBEC}

Nicole Morin Laurendeau *

Dans un souci d'améliorer la qualité et l'efficacité des interventions de l'agent de probation, le comité de formation et de perfectionnement du service de Probation du Québec a mis sur pied au printemps de 1977 un programme de perfectionnement clinique. Le contenu de ce programme a été élaboré à la suite d'un sondage effectué auprès de quarante-trois agents de probation ainsi que d'une analyse de dossiers de probation. Cette étude révèle, d'une part, une pratique peu structurée et une intervention qui va rarement au-delà du situationnel et, d'autre part, une référence quasi unique à la thérapie de la réalité de Glasser comme cadre théorique de l'intervention auprès des justiciables. Les agents de probation sont conscients de leurs limites en tant qu'intervenants; ils souhaitent élargir leurs possibilités d'intervention et réclament en particulier une formation concernant le mode d'intervention à court terme.

Grâce au programme de l'Université Laval, les membres du comité de formation et de perfectionnement ont été sensibilisés à un mode d'intervention à court terme. Il s'agit de la méthode de solution de problème The Art of Problem Solving, de Carkhuff (1973), psychologue américain, auteur de plusieurs livres sur des méthodes d'intervention en relation d'aide et fondateur de l'Institut HDR (Human Development Resource), à Amherst, Massachusetts. La méthode de solution de problème paraissant à première vue pertinente pour les agents de probation, les membres du comité ont organisé de concert avec l'université une session de formation pour une quinzaine d'agents recrutés à travers la province. Cette session d'une durée de quatre jours fut suivie de deux journées de rétroaction.

Des lectures préalables et conjointes au programme de formation sur la relation d'aide en général et sur l'œuvre de Carkhuff en particulier complétaient la formation pratique et fournissaient un cadre théorique intéressant.

* L'auteur est agent de probation dans le secteur nord du district de Montréal. Elle a une maîtrise en criminologie. 
Nous allons en premier lieu situer brièvement la méthode de solution de problème dans l'évolution spécifique de l'œuvre de Carkhuff, puis en faire la description et l'illustration. En second lieu, nous allons traiter de l'application de cette méthode au service de Probation et des réflexions personnelles et critiques qu'elle a suscitées ainsi que des perspectives d'avenir de l'expérience.

\section{CARKHUFF ET LA METHODE DE SOLUTION DE PROBLÈMES}

Robert R. Carkhuff est un chercheur qui, dans la ligne du pragmatisme américain, est soucieux de la rentabilité et de l'efficacité de la relation d'aide. Ses études faites en collaboration avec d'autres chercheurs dont B.G. Berenson, C.B. Truax et M. Mitchell mettent en relief le fait qu'une intervention peut non seulement être inefficace mais nuisible à un client, et en cela le professionnel n'est pas plus favorisé que le non professionnel. C.B. Truax affirme même qu'un conseiller sur trois est compétent en relation d'aide!

Compte tenu de l'urgence d'améliorer l'intervention des agents conseillers, Carkhuff développe de façon systématique les éléments de base nécessaires à la relation d'aide et met sur pied des outils en l'occurrence des échelles destinées à le mesurer. Par la suite, il développe également les diverses méthodes d'intervention qui présupposent une formation en relation d'aide. Ces méthodes d'intervention couvrent les différentes sphères de l'activité humaine tel le développement physique, intellectuel, émotionnel, etc. En fait, Carkhuff s'oriente vers une technologie humaine dont le seul critère est le mieux être individuel et collectif, soit l'efficacité.

En 1972, Carkhuff a eu l'occasion d'expérimenter dans une recherche une de ses méthodes d'intervention soit la méthode de solution de problème et de la comparer à d'autres modes d'intervention. Les données de cette recherche sont extrêmement intéressantes ${ }^{3}$ et il nous semble pertinent de résumer ici les conclusions de cette étude qui indiquent les critères d'efficacité en relation d'aide. Parmi ces conclusions, nous avons retenu les suivantes :

1. Pour détails, voir la revue Counseling Psychologist, 1972, 3 (3), p. $12-29$. 
un agent qui prend le temps de recevoir un client devient plus efficace. Dans l'expérience, on a doublé le taux de succès de $13 \%$ à $25 \%$ en passant d'entrevies de 5 à 10 minutes à des entrevues de 50 minutes;

un bon programme est plus utile qu'un conseiller formé de façon traditionnelle (taux d'efficacité de 50\%);

un bon programme joint à un conseiller spécialisé est encore plus efficace (taux d'efficacité de 67\%);

une formation en relation d'aide (attitudes de base en entrevue) engendre un très haut taux de succès $(91 \%)$.

En résumé, un intervenant, ayant reçu une formation en relation d'aide, qui applique un programme d'intervention structuré et adéquat, atteint le plus haut taux d'efficacité dans une relation d'aide.

Le résultat de la recherche témoigne entre autres de façon hautement significative de l'importance des attitudes de base en relation d'aide. Quelles sont ces attitudes de base que doit démontrer tout conseiller en entrevue ?

Carkhuff les divise en deux groupes : les qualités réceptives qui facilitent l'implication du client dans la relation d'aide, soient l'empathie, l'acceptation inconditionnelle (chaleur et respect) et l'authenticité : les qualités actives telles que la spécificité, la réaction immédiate (l'ici et le maintenant) et la confrontation qui complètent les qualités réceptives et qui orientent le client vers des modifications de comportement. On doit se référer au livre de L. Auger (1972), Communication et épanouissement personnel, pour une définition complète de chacune de ces qualités et pour une illustration des échelles de Carkhuff, graduées de 1 à 5 , qui permettent de mesurer chez un intervenant le degré de présence de chacune de ces qualités. Ainsi, pour ne pas être nuisible en relation d'aide, l'intervenant doit se situer au niveau 3. Les niveaux 4 et 5 lui permettent une intervention utile et positive. Les niveaux 1 et 2 risquent d'engendrer une détérioration chez le client ; or, selon C.B. Truax (1965), 40\% des intervenants se situent aux niveaux 1 et 2 .

Doté d'une formation sur ces qualités de base en entrevue, l'agent l'utilise par la suite à l'intérieur d'un programme d'intervention efficace. C'est ici que se situe la méthode de solution de problème, méthode d'intervention qui ne s'adresse pas spécifiquement à une clientèle délinquante. Visant la solution de pro- 
blème en général, elle parât adaptable à différents types de clientèle. En fait, Carkhuff divise en huit étapes (une entrevue hebdomadaire par étape) le processus naturel que chacun emploie inconsciemment lorsqu'il fait face à un problème (exploration, compréhension, action). Ainsi, cette méthode amène le client à cerner lui-même la zone problématique, à la définir de façon concrète, à inventorier les moyens de solution et à faire son choix sur un plan concret de l'action. Cette méthode ne vise pas à toucher à la dynamique profonde de la personnalité, mais permet de libérer des énergies en solutionnant un problème concret par des moyens concrets. De plus, par cet apprentissage, Carkhuff espère faciliter au client la possibilité de résoudre ses problèmes éventuels.

Le rationnel de Carkhuff en ce qui a trait au court terme apparaît peu élaboré. Néanmoins, la méthode de solution de problèmes répond à trois conditions : l'établissement de contrat, l'intervention sur une période limitée et la participation active du conseiller et du client, trois facteurs implicites aux méthodes dites de court terme.

Nous décrirons la méthode de solution de problèmes de Carkhuff en l'illustrant d'un exemple d'application intégrale à un client du service de Probation. Quelques données de l'histoire sociale du sujet paraissent toutefois nécessaires avant d'aborder la méthode comme telle.

Le justiciable Luc... ${ }^{2}$ s'était rendu coupable de vol qualifié et d'enlèvement. Son degré de criminalisation était inversement proportionnel à la gravité des agirs car si les actes posés étaient violents en soi, l'attitude du sujet durant la commission des délits était peu menaçante et teintée d'amateurisme. En fait, toute la dynamique du délit était reliée au fait qu'il était un parieur. Il était issu d'un milieu familial marqué par cette marginalité. L'argent revêtait pour lui une grande importance et était le seul moyen de se valoriser auprès des autres. Âgé de 28 ans, marié et père de trois enfants, il aurait voulu donner à sa famille des conditions de vie bien au-delà de celles que pouvaient lui assurer son salaire. Il essaie de jouer, accumule $\$ 15000$ de dettes et voit sa femme le quitter. Il s'ensuivit une désorganisation totale de son existence (perte d'emploi, dépression, etc.) et c'est

2. Pour des raisons de confidentialite, le nom a tete change. 
durant cette période qu'eut lieu le délit. Ce délit a également été commis à titre de chantage envers sa femme car il espérait ainsi lui communiquer son désespoir, afin qu'elle reprenne la vie commune ce qu'il a réussi d'ailleurs. Il n'en reste pas moins qu'au moment du délit et précédemment, la communication entre les époux était quasiment inexistante et leur vie conjugale marquée par des tensions qui n'ont fait qu'accentuer sa propension au jeu.

L'impact du processus judiciaire et le retour non définitif de son épouse ont obligé le client à un changement de comportement. Il requit de l'aide et une application de la méthode de Carkhuff paraissait pertinente. En voici l'illustration :

Phase I : Développement du problème

Etape I : exploration du problème

Etape II : compréhension du problème
1) faciliter l'exploration et répondre au comportement du client ;

1) utiliser sa propre expérience, se faire une image du client.

Luc... précise son objectif, il veut cesser de jouer aux courses. Il faut l'aider à définir ce qui le pousse à jouer aux courses et travailler sur ce matériel. En guise d'amorce au problème, il lui est demandé de faire part des situations où il décide de se rendre aux courses. Il inscrit au tableau :

- quand je suis tout seul ;

- quand je n'ai pas de loisirs ;

— quand ça va mal à la maison ;

- quand je ne suis pas content de moi ;

- quand les autres ne me font pas confiance ;

- quand je n'ai pas d'argent (pas capable de faire un budget);

- quand je veux donner de l'argent à Lisette (femme du sujet) et aux enfants ;

- quand je suis découragé ;

- quand je ne me sens pas un homme.

D'autre part, l'exploration exige de faire élaborer Luc sur $\propto$ que signifie l'argent pour lui. Il le traduit ainsi :

- argent $=$ me faire aimer par Lisette et les enfants ; 
- argent $=$ exister pour les autres ;

— argent $=$ être un homme ;

- argent $=$ ne pas être petit ;

- argent $=$ avoir confiance en moi.

En effet, le sujet n'arrive pas à côtoyer les autres individus quand il n'a pas suffisamment d'argent. S'il est dans l'ascenseur sans argent, il sort dès qu'une personne entre car il se sent écrasé. Lors des visites de sa belle-famille à la maison, il s'isole dans une pièce voisine parce qu'il ne peut montrer, par son ameublement, une certaine réussite. Il se considère comme trop petit par rapport aux autres tout en souhaitant se sentir à un niveau d'égalité. Lorsqu'il se trouve seul ou lorsqu'il est en situation de conflits, ce sentiment d'infériorité est mis à vif. Les courses représentent pour lui une sorte de magie : gagner signifierait être capable d'exister aux yeux des autres.

\begin{tabular}{|c|c|c|}
\hline $\begin{array}{l}\text { Etape III : définition } \\
\text { du problème }\end{array}$ & $\begin{array}{l}\text { 1) } \\
\text { 2) }\end{array}$ & $\begin{array}{l}\text { en donner une définition } \\
\text { spécifique ; } \\
\text { le poser en termes de re- } \\
\text { lations mathématiques } \\
\text { simples }(+,-,=,>\text {, } \\
<) \text {. }\end{array}$ \\
\hline $\begin{array}{c}\text { Etape IV : définition } \\
\text { du but }\end{array}$ & $\begin{array}{l}\text { 1) } \\
\text { 2) } \\
\text { 3) }\end{array}$ & $\begin{array}{l}\text { définir les buts en ter- } \\
\text { mes spécifiques; } \\
\text { poser les buts en termes } \\
\text { de relations mathémati- } \\
\text { ques simples; } \\
\text { transformer le problème } \\
\text { en but. }\end{array}$ \\
\hline
\end{tabular}

Il apparaît qu'un fort sentiment d'infériorité est sousjacent et qu'il constitue un bon objectif de travail. Dans le langage du sujet, il veut * grandir >, il ne veut plus se sentir * un petit chien * à côté d'autrui, il veut cêtre quelqu'un », il veut \& prendre confiance en lui ?.

L'objectif est encore trop vaste. Il doit préciser devant qui il se sent petit et comment cela se manifeste. La relation de Luc... avec son épouse paraît alors problématique. Luc... prend conscience du fait qu'elle l'écrase parce qu'il n'arrive jamais à lui dire ce qu'il pense. Lisette décide de tout et s'il tente de 
s'exprimer il le fait maladroitement. De vives discussions s'ensuivent et conséquemment Luc... prend le chemin de l'hippodrome. Ce rituel est bien installé; sa femme ne lui fait donc guère confiance et le domine davantage; c'est le cercle vicieux. L'objectif devient ainsi plus précis. Luc... veut apprendre à échanger avec Lisette, c'est-à-dire trouver des occasions propices à l'échange. Le problème (difficulté d'échanger avec Lisette) se transforme en but (trouver des occasions pour favoriser l'échange). En termes de relations mathématiques simples, cela s'exprime ainsi : les occasions propices d'échange ne sont pas assez nombreuses $(<)$, il faut les augmenter $(>)$.

Phase III : Examen des voies de solution

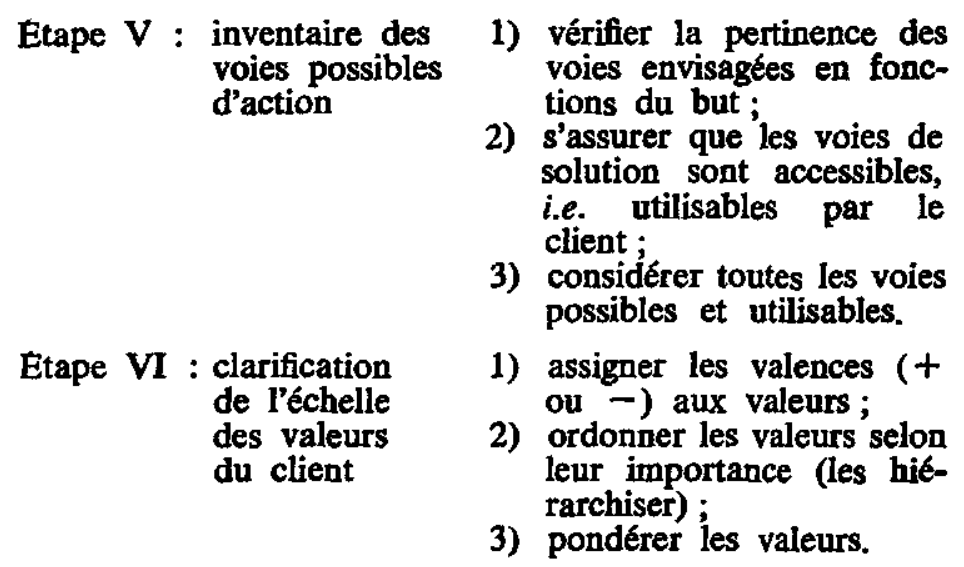
d'action :

Lors de l'entrevue de l'étape $V$, Luc... énumère six moyens

- passer une fin de semaine seul avec Lisette;

- profiter des ballades en voiture ;

- rencontrer des couples d'amis et échanger avec eux ;

- se rencontrer en fin de soirée, après une sortie;

- participer à des fins de semaine pour couples;

- participer à des sessions (une soirée par semaine) pour couples.

Luc... semble avoir besoin de l'appui et de l'exemple des autres. Une insécurité fondamentale lui fait souhaiter de se modeler sur un autre couple. 
L'étape des valeurs (VI) est initiée sous l'étiquette c la vie en rose avec Lisette \$. Pour ce type de client, elle est tout de même l'étape la moins accessible. Malgré les références au rêve, à la fantaisie, il est difficilement capable d'établir ses propres valeurs. Etant très peu conscient de ses besoins, il se réfère constamment aux autres, surtout à sa femme, pour définir ce qui est important pour lui. Avec beaucoup de présence et de vigilance de la part du conseiller, il établit après deux entrevues cette liste hiérarchisée et pondérée :

- être capable d'affronter Lisette $(+10)$;

- dire ce que je ressens $(+9)$;

- avoir la confiance de Lisette (+9);

- prendre le temps de se parler et de s'écouter $(+8)$;

- être calme avec Lisette $(+8)$;

- prendre des décisions avec Lisette $(+7.5)$;

- penser avant de parler et d'agir $(+6)$;

- rendre service à Lisette $(+2)$;

- faire plaisir à Lisette $(+2)$;

- me chamailler avec Lisette $(+2)$.

Phase IV : Choix et développement des moyens d'action

Etape VII : Choix des moyens d'action
1) voir si les moyens d'action (un à un) favorisent le développement ('actualisation) de chacune des valeurs;

2) déterminer à quel degré tel moyen d'action actualise telle valeur;

3) multiplier la valence et la pondération pour chacune des valeurs en regard de chacun des moyens d'action ;

4) faire la somme algébrique des valeurs accordés à chaque moyen d'action. 
$\begin{aligned} \text { Etape VIII : } & \text { élaboration d'un } \\ & \text { programme d'action } \\ & \text { (mise en application } \\ & \text { du moyen choisi) }\end{aligned}$
1) choisir un pro- gramme d'action;
2) mettre le program- me en application.

A l'étape VII, il s'agit de confronter maintenant la liste des valeurs avec chacun des moyens d'action. Suite au travail accompli à l'étape des valeurs, le sujet fait une révision des moyens d'action et apporte quelques changements. Le tableau qui suit résume la situation.

Il en ressort à l'étape VIII que quatre moyens d'action sont significatifs. En effet, selon Carkhuff, les moyens d'action qui totalisent un pourcentage de $70 \%$ et plus ont une grande chance de succès advenant leur mise en application. Dans ce cas-ci, le marriage encounter a été l'objectif choisi par le client car c'était le moyen d'action privilégié par son épouse et, à l'époque, des amis communs avaient favorisé leur inscription à cet organisme. Malheureusement, l'application de la méthode de solution de problème avait eu lieu en surveillance sous caution. Suite à la dernière entrevue, le sujet a été condamné à une courte période d'emprisonnement. La probation suite à cette période devrait permettre une reprise du processus.

Ceci est un exemple d'application intégrale de la méthode à un délinquant. La section suivante permettra de situer cet exemple dans l'ensemble de l'expérience de Carkhuft au service de Probation.

\section{OBSERVATIONS QUANT A L'EXPERIENCE DU PROGRAMME DE CARKHUFF AU SERVICE DE PROBATION}

Seize agents de probation avaient reçu en septembre 1977 une formation de quatre jours sur cette méthode, suivie de deux journées de rétroaction en février 1978. L'objectif visé consistait à utiliser cette technique avec des clients et, par la suite, à évaIuer sa possibilité d'application et son efficacité auprès d'une population délinquante. Malgré la formation reçue, les résultats escomptés n'ont pas été atteints. En effet, les deux journées de rétroaction ont permis de constater que la méthode a été rarement appliquée de façon intégrale. Des difficultés ont surgi au niveau de la sélection des clients et de la présentation de la méthode. Plusieurs agents n'arrivaient pas à motiver un client à se soumettre à ce mode d'intervention. Il est vrai qu'une trop 
Problème : Occasions propices pour échanger avec Lili $^{2}$ pas assez nombreuses $(<)$

But : $\quad$ Augmenter les occasions d'échange $(>)$

\section{MOYENS D'ACTION}

\begin{tabular}{|c|c|c|c|c|c|c|c|}
\hline Valeurs & & $\begin{array}{l}\text { Fin de } \\
\text { semaine } \\
\text { seul } \\
\text { avec Lili }\end{array}$ & $\begin{array}{l}\text { Ballade } \\
\text { d'auto }\end{array}$ & $\begin{array}{c}\text { Rencontre } \\
\text { avec } \\
\text { couple }\end{array}$ & $\begin{array}{l}\text { Marriage } \\
\text { encounter }\end{array}$ & $\begin{array}{c}\text { Soirées } \\
\text { organisées } \\
\text { pour } \\
\text { couples }\end{array}$ & $\begin{array}{c}\text { Période fixe } \\
\text { de } \\
\text { rencontre } \\
\text { à la maison }\end{array}$ \\
\hline Affronter Lili & $(+10)$ & $++(20)^{2}$ & $+(10)$ & $++(20)$ & $++(20)$ & $++(20)$ & $++(20)$ \\
\hline Dire ce que je ressens & $(+9)$ & $++(18)$ & (9) & $++(18)$ & $++(18)$ & $++(18)$ & $++(18)$ \\
\hline Avoir la confiance de Lili & $(+9)$ & (0) & $(0)$ & $+\quad(9)$ & $\pm+(18)$ & $+t(18)$ & $+\quad(9)$ \\
\hline Prendre le temps de s'écouter & $(+8)$ & $+\quad(8)$ & $t-(0)$ & $+\quad(8)$ & $++(16)$ & $++(16)$ & $++(16)$ \\
\hline Être calme avec Lili & $(+8)$ & $++(16)$ & $+\quad(8)$ & $+t(16)$ & $+\quad(8)$ & $t+(16)$ & (8) \\
\hline Prendre des décisions avec Lili & $(+7,5)$ & (0) & $(0)$ & $+(7,5)$ & $+(7,5)$ & $+(7,5)$ & $++(15)$ \\
\hline Penser avant d'agir & $(+6)$ & $(0)$ & (0) & $+\quad(6)$ & $+\quad(6)$ & $++(12)$ & $++(12)$ \\
\hline Rende service à Lili & $(+2)$ & $(0)$ & (0) & (0) & $(0)$ & $0 \quad(0)$ & $0 \quad(0)$ \\
\hline Faire plaisir à Lili & $(+2)$ & (4) & (2) & $+t(4)$ & $++(4)$ & (4) & $++(4)$ \\
\hline Se chamailler avec $\mathrm{Lili}$ & $(+2)$ & $++(4)$ & $(0)$ & (0) & (0) & $0 \quad(0)$ & (0) \\
\hline $127^{8}$ & & 70 & 29 & $\begin{array}{l}88,5 \\
70 \%\end{array}$ & $\begin{array}{l}97,5 \\
76,7 \%\end{array}$ & $\begin{array}{c}111,5 \\
87,7 \%\end{array}$ & $\begin{array}{c}102 \\
80,3 \%\end{array}$ \\
\hline
\end{tabular}

1. Il convient de signaler qu'il s'agit d'un nom fictif et que le tableau traduit le langage du client.

2. Le client a accordé une pondération à chaque valeur. Un moyen d'action peut favoriser un peu $(t)$, beaucoup $(t+)$ l'actualisation de cette valeur, il peut nuire un peu $(-)$, beaucup $(--)$, il peut aider sous certains aspects et nuire sous d'autres $(+-)$ ou encore n'avoir aucun rapport avec la valeur (0). Le chiffre qui suit ces signes est le produit de la multiplication entre la pondération des moyens d'action et le poids accordé aux valeurs.

3. Le maximum de points que peut donner un moyen d'action est obtenu par la multiplication du poids de chaque valeur par le nombre de signes positifs $(+)$. Ce total sert de bareme pour fixer le pourcetage de succes de chaque moyen d’action. 
grande préoccupation et insistance à vouloir appliquer la méthode a pu nuire aux attitudes thérapeutiques et a peu favorisé la motivation et la disponibilité du client. D'autre part, le type même de la clientèle du service de Probation et la sélection aléatoire établie au départ ont pu rendre l'utilisation de la méthode difficile. En effet, certains justiciables ont démontré souvent une faible capacité d'introspection; ils se présentaient en réponse à une obligation légale, ce qui nuisait à leur motivation à solutionner leurs problèmes - condition pré-requise à l'application de la méthode. D'autres sujets présentaient des problèmes d'ordre psychiatrique qui nécessitaient une intervention plus spécialisée et beaucoup plus longue. Les vrais délinquants, pour leur part, étaient difficilement accessibles à cette forme d'aide. Ceci limitait donc les possibilités d'application et engendrait souvent des échecs.

Par contre, les obstacles à l'utilisation de la méthode ne se situaient pas uniquement du côté de la clientèle. Les agents de probation ont relevé chez eux un certain manque de compétence et de formation au niveau des attitudes de base en relation d'aide, de là leur insécurité dans l'utilisation d'une nouvelle méthode. Ils ont fait part également de leur difficulté à structurer leur intervention, habitués qu'ils étaient à une pratique quelque peu décousue. Enfin, les contraintes telles que le volume et le style de travail (situations d'urgence, etc.) ainsi que l'isolement de certains agents dans des régions rurales ont diminué la motivation de quelques-uns. Au-delà de ces deux catégories d'obstacles, un manque de soutien et de supervision dans chaque région au cours de l'expérimentation a engendré une certaine démobilisation chez les agents ou, tout au moins, ne leur a pas fourni la stimulation nécessaire.

Les bénéfices secondaires retirés de ce programme de formation étaient cependant importants. Les agents ont pris conscience de la nécessité de structurer et de rentabiliser leurs interventions, du besoin de susciter et de mobiliser la motivation du client au changement et à la résolution de ses problèmes et ce, en dépit du rôle de contrôle inhérent à leur tâche. Ces prises de conscience orientaient vers un changement très sensible de la pratique de la probation qui, jusque-là, s'avérait peu structurée et situationnelle. 
En résumé, des lacunes au niveau des outils de base en relation d'aide, un manque de supervision spécifique des expériences d'application et d'assimilation de la méthode ainsi que la nécessité de motiver au préalable le délinquant, semblaient des éléments importants qui ont nui à l'utilisation du modèle de Carkhuff par les agents de probation. L'évaluation du projet indiquait donc une certaine timidité dans l'expérimentation de la méthode et traduisait un écart considérable par rapport aux attentes des responsables.

Ces commentaires des agents faits lors des journées de rétroaction ont relégué au second plan les remarques sur la méthode en tant que telle et sur certains résultats obtenus. Il a été étonnant, en effet, de constater que plusieurs clients étaient disposés à se présenter chaque semaine, ce qui représentait l'immense avantage de les situer hors du cadre juridique et d'éprouver ainsi leur motivation. Les tentatives d'application intégrale ont permis à certains agents une assimilation de la méthode. Elles ont suscité aussi des interrogations et des commentaires sur la nécessité, par exemple, de modifier et d'inverser certaines étapes, vu les caractéristiques de la clientèle délinquante, sur la réaction des clients à certains éléments tels l'emploi des signes mathématiques et l'utilisation d'un tableau confirmant l'impact et limportance de ceux-ci. Il a été noté également chez les justiciables une évolution positive même s'il y eut interruption de la démarche; le justiciable semble retirer, du seul fait de son implication à résoudre son problème, une grande valorisation personnelle et une meilleure confiance en lui-même. $\AA$ ce stade-ci de l'expérimentation, il paraît difficile de préciser les types de clients susceptibles de bénéficier de ce mode d'intervention privilégié.

Ces succès et ces échecs ont engendré chez les agents impliqués beaucoup d'intérêt, de satisfaction et de curiosité face à la possibilité d'adapter la méthode de Carkhuff à la clientèle du service de Probation.

\section{RÉFLEXIONS CRITIQUES SUR L'EXPÉRIENCE}

Il est évident que cette expérimentation est limitée. Étant jointe à quelques années d'expérience auprès des délinquants, elle permet tout de même des réflexions critiques, tant sur la pertinence d'utiliser cette méthode au service de Probation, 
qu'au plan de la formation et de la sélection des agents de probation. Elle permet également d'envisager des perspectives d'avenir.

D'un point de vue théorique, la méthode de Carkhuff paraît pertinente pour une clientèle délinquante et ce, sous plusieurs aspects. Tout d'abord, les conditions préalables qu'elle exige en ce qui concerne les dimensions de base en relation d'aide, paraissent indispensables surtout à un agent de probation qui se doit de travailler au niveau de la motivation avant de penser à l'utilisation d'une méthode quelconque. En deuxième lieu, la structure même de la méthode, par sa rapidité, son orientation vers le concret plutôt que l'abstrait, sa simplicité et l'obligation d'impliquer le client dans la solution de son problème vers une meilleure adaptation sociale, répond à certains besoins typiques chez le délinquant. Le succès en tant qu'objectif visé, permet également de défaire par une action, si minime soit-elle, et au cours d'une période limitée, l'image négative que le délinquant a de lui-même. En outre, une analyse de dossiers nous autorise à croire que cette méthode serait accessible à un pourcentage intéressant de justiciables. Ne serait-elle applicable qu'à $10 \%$ de ceux-ci, il serait souhaitable dans l'optique d'un meilleur service à la clientèle que certains agents maîtrisent ce mode d'intervention. Enfin, la pertinence de ce mode d'intervention pour le service de Probation ressort singulièrement si l'on se réfère à d'autres techniques telles la « gestalt », l'analyse transactionnelle, etc. En effet, ces modes d'intervention impliquent souvent des modifications dans la structure de la personnalité et deviennent difficilement applicables de façon courante dans les services gouvernementaux soit parce que cela exige une orientation particulière du thérapeute, ou encore beaucoup de temps à consacrer à leur application.

$\mathrm{Au}$ niveau de la formation et de la sélection des agents de probation, l'expérience aura mis en relief, chez les criminologues en particulier, une formation universitaire plutôt académique et une certaine faiblesse au plan clinique. L'apport de Carkhuff, tant par sa formation systématique sur les dimensions de base en relation d'aide que par ses méthodes d'intervention, pourrait constituer un atout important dans une formation universitaire pour ceux qui s'orientent vers l'aspect clinique de la profession. Quant à la sélection des agents de probation, si 
la vérification et l'assimilation des connaissances acquises sont des critères de base privilégiés par les membres d'un jury de sélection, une attention devrait également être accordée aux aptitudes proprement cliniques du candidat. Ainsi, l'agent de probation serait également à l'aise dans ses deux fonctions, soit la production de rapports présentenciels et la surveillance dans une optique de réhabilitation.

Dans l'exercice de son rôle, l'agent de probation souligne fréquemment la dualité * contrôle versus thérapie ». Pour lui, les contraintes légales sont souvent en contradiction avec une ambiance thérapeutique. L'expérience de Carkhuff indique que, dans plusieurs cas, il est possible, grâce aux outils de base en relation d'aide, d'allier ces deux aspects quelque peu contradictoires. Pour Carkhuff, ce sont des individus qui motivent d'autres individus et les qualités interpersonnelles en relation d'aide offrent un éventail de solutions au problème de la motivation. L'utilisation des qualités tant réceptives qu'actives, en entrevue avec un délinquant, permet à l'agent de préciser de façon adéquate son rôle et le cadre dans lequel se déroulera l'intervention. Ceci lui permet également d'être sensible aux sentiments de frustration, d'anxiété ou à tout autre sentiment négatif du client vis-à-vis de la probation. Une fois les rôles clarifiés et les résistances liquidées, un justiciable pourra être motivé et acceptera d'établir à l'intérieur des limites légales un contrat s'il perçoit qu'il en est le bénéficiaire. Il est intéressant également de constater pour l'agent de probation, qui se réfère à la thérapie de la réalité de Glasser dans l'exercice de son rôle, que ces deux auteurs se rejoignent sensiblement dans leur philosophie. Carkhuff complète cependant Glasser par la mise sur pied d'un matériel plus concret de travail. Ce matériel de travail se situe au niveau du lien thérapeutique que Carkhuff définit et mesure à l'aide de ses échelles, ainsi qu'au niveau de l'apprentissage et du changement que Carkhuft traduit par des méthodes bien structurées telle la méthode de solution de problèmes. Carkhuff dépasse également Glasser par ses études sur la confrontation. Comme lui, il admet que cette dernière ne peut être utilisée sans le lien thérapeutique. Même si la confrontation est un thème bien en vogue, elle doit être selon ses dires, utilisée parcimonieusement. Pour lui, elle n'est jamais nécessaire et suffisante en soi. Accompagnée des dimensions de 
base en relation d'aide, elle peut cependant être efficace et utile. Il est bon pour un agent de probation de se rappeler ces mises en garde car le comportement de plusieurs délinquants incite souvent à la confrontation qui apparaît parfois trop tôt dans les interventions de l'agent de probation.

En résumé, l'expérience de la méthode de Carkhuff permet de constater que le rôle de l'agent de probation repose avant tout, quel que soit l'instrument utilisé, sur les qualités interpersonnelles en relation d'aide. Indépendamment des questions de confrontation, de dualité de rôles, de pertinence de méthodes de traitement, les qualités interpersonnelles demeurent toujours un outil indispensable et la base même de l'efficacité.

Au terme de cette expérience, j’ai acquis une connaissance de Carkhuff, de sa philosophie, de ses objectifs et de ses programmes d'interventions. Je maintiens un intérêt particulier pour son cuvre en fonction des besoins de l'agent de probation. Par contre, l'expérience vécue se situant au niveau d'une recherche exploratoire n'a pas été assez poussée pour répondre à l'interrogation sur l'applicabilité ou l'adaptation de la méthode de solution de problèmes à la clientèle du service de Probation. Or, la réponse à cette interrogation semble essentielle non seulement pour rentabiliser une expérience déjà coateuse, pour permettre à certains agents de poursuivre le processus amorcé et d'exploiter au maximum leur potentiel, mais aussi pour répondre plus adéquatement à la clientèle, ce qui en définitive est la raison d'être d'un service.

Cette interrogation milite donc en faveur de la poursuite du programme; l'expérience acquise nous oriente alors vers des propositions concrètes pour l'avenir et peut également laisser poindre des hypothèses cliniques. Au niveau des propositions concrètes, il apparaît d'abord que ce mode d'intervention est plus accessible en milieu urbain qu'en milieu rural. Ne serait-ce qu'en considérant la distance, cette méthode devient inapplicable pour plusieurs en milieu rural. Se déplacer sur un long parcours toutes les semaines représente en temps et en argent un coût élevé et la plupart des justiciables sont issus d'un milieu défavorisé. Ce coût est le même pour le service de Probation advenant que l'agent doive lui-même se déplacer. L'isolement du milieu rural apporte également le même élément de pénalité face à l'entraînement et à la formation. Ils n'ont pas l'équipement 
audiovisuel et leur déplacement vers les zones urbaines entraîne des frais beaucoup trop élevés.

D'autre part, souhaiter que tous les agents de probation maîtrisent l'emploi de ce mode d'intervention serait quelque peu utopique. Si cette méthode peut être bénéfique à 10 ou $20 \%$ de la clientèle, un agent desservant un ou deux lieux de travail pourrait suffire à répondre aux besoins des clients. L'expérience renseignera davantage mais il semble que ce soit réaliste comme suggestion de départ. Pour les agents qui seront disponibles à l'utilisation de cette méthode, il faudra prévoir une revision de leur somme de travail car l'énergie et le temps nécessaire pour ce mode d'intervention ne sont nullement comparables avec une surveillance traditionnelle.

Il faudra fournir également aux agents de probation des conditions physiques propices à l'expérimentation. Ainsi, l'agent qui aura à travailler huit semaines consécutives avec un client en entrevues d'environ deux heures ne pourra pas fonctionner à l'intérieur d'un aménagement dit paysager ${ }^{3}$. Sans pousser à l'exagération, le cadre physique présente néanmoins une certaine importance et peut faire partie des conditions propices. Il est déjà assez difficile d'établir une relation d'aide sans risquer de la compromettre par un environnement physique inadéquat.

Sur le programme comme tel, cette deuxième étape de la recherche exploratoire devrait en être une de supervision directe quelle qu'en soit la forme. Une centaine d'heures de supervision en situation de laboratoire (jeux de rôle, bandes audiovisuelles, enregistrements), sous l'œil averti d'un superviseur paraît une modalité souhaitable et réalisable. Trois agents sont présentement intéressés à cette forme de supervision et le service de Probation offre tout l'équipement nécessaire.

Le contenu de ce programme de supervision devrait revêtir un double objectif ; il devrait d'abord permettre à l'agent de se situer sur l'échelle de Carkhuff en fonction des dimensions de base en entrevue et évoluer à partir de ces données. $\AA$ l'encontre de cette première étape de la recherche exploratoire qui prenait pour acquise la compétence des agents dans ces dimensions de base, la seconde étape devrait consacrer, vu les lacunes

3. Il s'agit de surfaces à bureaux non divisées par des cloisons complètes où $\mathrm{il}$ est impossible de s'isoler derrière les portes fermées. 
relevés, au moins $60 \%$ de son programme sur l'acquisition de ces attitudes fondamentales en entrevue. Une compétence accrue en ce domaine permettrait ainsi à l'agent de probation d'éviter les échecs rencontrés à cette première étape. Un deuxième objectif de ce programme serait de développer une habileté dans l'application ou l'adaptation de la méthode de solution de problèmes et ce n'est qu'à ce niveau que pourraient être formulées certaines hypothèses cliniques sur les types de délinquants plus susceptibles de bénéficier de ce mode d'intervention.

Déjà intuitivement, en référence à la typologie de Sullivan, Grant et Grant, typologie très utilisée présentement et orientée vers le traitement, il apparaît que la méthode de solution de problème ne serait accessible que pour les délinquants qui présentent un minimum de maturité interpersonnelle. Ainsi les individus du niveau $A$ de cette typologie (asociaux passifs et agressifs) seraient peu aptes à bénéficier du processus de Carkhuff vu certaines caractéristiques telles leur incapacité d'autocritique, leur absence d'attitudes actives face à la vie, leur forte incidence de pensées magiques, etc. Les individus du niveau $B$ seraient déjà plus accessibles à cause de leur besoin de pensées concrètes et de leur capacité de planification de courte durée, les individus du niveau $C$ faisant preuve d'une plus grande capacité d'introspection et de motivation devraient bénéficier avantageusement d'un tel mode d'intervention.

L'expérience clinique, d'autre part, laisse deviner certaines catégories de clients à qui ce mode d'intervention pourrait être proposé tout en espérant un taux significaif de réussite. Il en est ainsi des probationnaires aux prises avec des problèmes de relations interpersonnelles tels que la gêne, la timidite, l'absence de relations sociales, le rejet social, l'incapacité d'établir une relation affective, les problèmes conjugaux, etc., ou encore des problèmes au niveau du travail comme l'instabilité, l'orientation, la recherche d'emploi. La méthode de solution des problèmes pourrait également venir compléter le soutien de groupe que reçoivent souvent les alcooliques et les drogués. Par contre, les délinquants qui présentent des problèmes psychiatriques paraissent devoir être écartés de ce mode d'intervention.

Toutes ces hypothèses, dans une seconde étape de la recherche exploratoire, devraient être formulées et vérifiées. Elles permettraient d'apporter ainsi une réponse tant sur l'applicabilité 
que sur l'efficacité de cette méthode de Carkhuff pour les agents de probation. Il y a place ici pour beaucoup de créativité et d'optimisme et les agents de probation ont vivement manifesté le besoin de maîtriser un autre mode d'intervention.

L'atteinte de ces deux objectifs du programme de formation permettrait par la suite aux agents supervisés de faire partager leur compétence à l'ensemble du personnel du service de Probation. Cette formation acquise pourrait être exploité par le service de diverses façons. Ainsi, les agents formés pourraient se servir des enregistrements audiovisuels produits jusqu'ici comme matériel didactique et pédagogique. Ils pourraient également, à l'intérieur des plans annuels de formation et de perfectionnement, concevoir, élaborer et administrer des programmes de formation sur les attitudes de base à partir des échelles de Carkhuff puis, sur la méthode de solution de problèmes en relation avec certaines catégories de clients. Enfin, ils pourraient être utilisés pour les programmes concernant les nouveaux employés surtout en termes de supervision dans leur rôle de surveillance.

Ces perspectives d'avenir paraissent rélistes et portent à croire en la nécessité de poursuivre cette recherche exploratoire sur la méthode de Carkhuff. Si cette dernière s'avère une réussite, il est permis d'espérer qu'en appliquant chez une partie de la clientèle la méthode de solution de problème, il en résultera une meilleure adaptation sociale et ce qui préviendra ainsi le recours à d'autres organismes sociaux ou le retour dans le système judiciaire. De cette façon, les frais initiaux de ce programme seront alors largement récupérés. 


\section{BIBLIOGRAPHIE}

ANKERSMITH, E. (1976) : - Setting the contract in probation 3, Federal Probation, juin, p. 28-33.

ASPY, D.N. and F.N. ROEBUCK (1977) : Kid's don't learn from people they don't like, Amherst, Massachusetts, Human Resource Development Press.

AUGER, L. (1972) : Communication et epanouissement personnel, Editions de l'Homme, Ottawa.

BERENSON, B.G. (1975) : Belly to belly, back to back : the militant humanism of R.R. Carkhuff, Amherst, Massachusetts, Human Resource Development Press.

BERENSON, B.G. and K. MITCHELL (1974) : Confrontation for better or worse, Amherst, Massachusetts, Human Resource Development Press.

BLAKEMAN, J., R.R. CARKHUFF, T. KELLING and O. PIERCE (1977) : Interpersonal communication skills for correctional management, Carkhuff institute of human technology, Amherst, Massachusetts, Human Resource Development Press.

CAREY-BÉLANGER, E. (1974a) : Intervention planifice à court terme s, Service social, Universite de Laval, 23, 2-3, 6-11.

CAREY-BELANGER, E. (1974b) : * Étude critique de recherches poursuivies sur le traitement planific à court terme en Service social personnel », Service social, Université de Laval, 23, 2-3, 85-108.

CARKHUFF, R.R. (1967) : Beyond Counseling and Therapy, New York, Holt, Rinehart and Winston, 2nd Edition.

CARKHUFF, R.R. (1969a) : Helping and Human Relations : selection and training, New York, Holt, Rinehart and Winston.

CARKHUFF, R.R. (1969b) : Helping and Human Relations : practice and research, New York, Holt, Rinehart and Winston.

CARKHUFF, R.R. (1971) : The development of human resources, educational, psychology and social change, New York, Holt, Rinehart and Winston.

CARKHUFF, R.R. (1972a) : The development of systematic human resource development models \$, The Counseling Psychologist, 3 (3), 4-11.

CARKHUFF, R.R. (1972b) : - New directions in training for the belping professions : toward a technology for human and community resource development *, The Counseling Psychologist, 3 (3), 12-29.

CARKHUFF, R.R. (1972c) : The art of helping. A guide for developing helping skills for parents, teachers and counselors, Amherst, Massachusetts, Human Resource Development Press.

CARKHUFF, R.R. (1973): The art of problem-solving. A guide for developing problem-solving skills for parents, teachers, counselors and administrators, Amherst, Massachusetts, Human Resource Development Press.

CARKHUFF, R.R. (1974) : How to help yourself. The art of program development, Amherst, Massachusetts, Human Resource Development Press. 
CARKHUFF, R.R. and G.B. BERENSON (1976) : Teaching as treatment, Amherst, Massachusetts, Human Resource Development Press.

CARKHUFF, R.R. (1977) : The art of helping III, Massachusetts, Human Resource Development Press.

COOTE, C. (1974) : Q Quelques considérations sur l'intervention planifice a court terme ", Service social, Université de Laval, 23, (2-3), 11-25.

DAUNAIS, J.P. (1974) : Méthodes de psychotherapies brèves *, Courrier-Cours, Section clinique, 1, (3), 1-21.

GLASSER, W. (1971) : La \& Reality Therapy \&, nouvelle approche therapeutique par le rtel, Paris, Epi.

HALMOS, P. (1965) : The faith of the counsellors, Constable-London.

KRUMBOLTZ, J.D. and C.E. THORENSEN (1969) : Behavioral Counseling, case and techniques, New York, Holt, Rinchard and Winston.

LAREAU-BÉLANGER, J. (1974) : Le traitement dans lintervention planifie a court terme $*$, Service social, $23(2-3), 45-57$.

MAC DOUGALI, C. (1978) : - A study of the effectiveness of the e timelimited task oriented a social work counseling approsech *, The social worker, mars 127-130.

MAILLOUX, N. (1965) : - Délinquance et répetition compulsive *, Contributions a l'étude des Sciences de lhomme, Centre de recherches en relations humaines, Montreal.

MUCCHIELLI, R. (1974) : Comment ils deviennent delinquants, Editions sociales françaises.

MUCCHIELLI, R. (1977) : L'entretien face d face de la relation d'aide. Paris, Entreprise moderne d'éditions, Editions ESI.

ROCHER, G. (1968) : Introduction d la sociologie générale, vol. L, Montreal, Editions HMH.

ROGERS, C. (1967) : Le développement de la personne humaine, Paris, Dunod.

ROGRRS, C. (1972) : Liberté pour apprendre, Paris, Dunod.

SAINT-ARNAUD, Y. (1972) : La personne humaine, Montrtal, Editions de l'Homme.

TRUAX, C.B., MTCHELL, K. (1965) : Research on certain therapist interpersonal skills in relation to process and outcome, $\boldsymbol{H}$ and book of clinical psychology, Editions Wolhman B.B.

WEISMAN, I. (1967) : Offender status, role behavior, and treatment considerations, Social casework (juillet), 422-425.

WOLBERG, L. (1965) : Short-term psychotherapy, New York, Grum and Shatton. 